Mots. Les langages du politique

Suisse, laboratoire politique européen ?

\title{
Professions de foi et élection présidentielle de 2002. Une approche verbale de la distance entre candidats
}

Jean-Michel Rampon

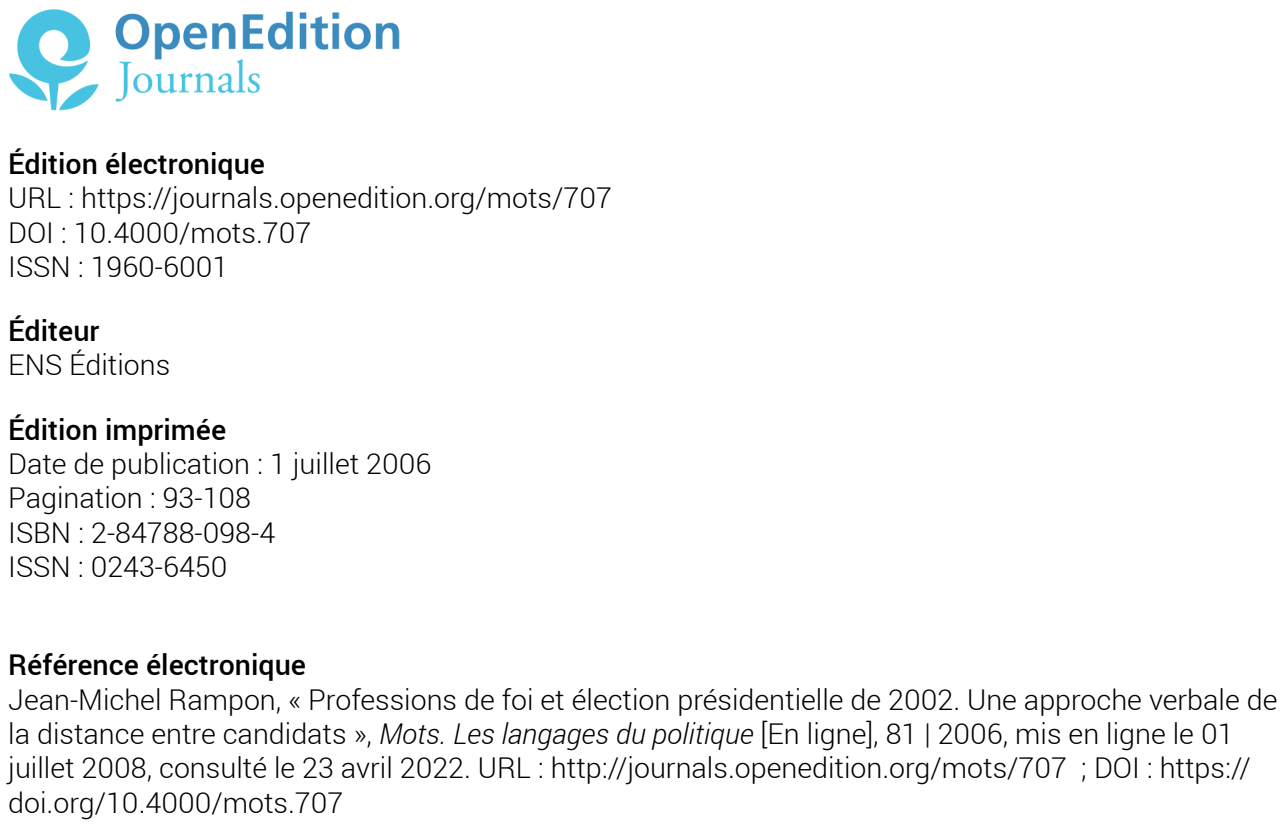

(c) ENS Éditions 


\section{Professions de foi et élection présidentielle de 2002. Une approche verbale de la distance entre candidats}

À ce jour, il existe au moins deux études qui portent sur les professions de foi de l'élection présidentielle française de 2002. La première, menée par Marc Bonhomme (2004), a consisté à soumettre le positionnement énonciatif des professions de foi au double registre monologique et dialogique pour, in fine, en apprécier au plus près les différentes gradations. Un autre auteur, Hugues Constantin de Chanay ${ }^{1}$, a pris le parti - et d'une certaine manière le pari - de faire passer un test auprès de publics diversifiés à partir de ce qu'il appelle les «prospectus » des candidats à l'élection présidentielle de 2002 :

Pourquoi un test? Pour vérifier une intuition éprouvée à la première lecture de ces prospectus de profession de foi, à l'époque où ils étaient d'actualité, avec leur fonction d'appel au vote: celle que beaucoup d'énoncés étaient comme interchangeables, n'appartenant en propre à aucun discours. (Constantin de Chanay, 2004, p. 1)

Le propos revient ici à se demander jusqu'à quel point le genre de discours délibératif justement associé à ces productions discursives (permettre à l'électeur de se décider quant à ses intentions de vote) n'est pas contrarié par le recours à la dimension épidictique de mise en valeur du candidat (dimension du reste attestée par l'analyse de M. Bonhomme). Au moment de faire le bilan du test qu'il a engagé, $\mathrm{H}$. Constantin de Chanay fait le constat suivant :

Malgré une tendance à la décoloration, en particulier lorsque l'on fait une lecture «superficielle» (se cantonnant par exemple aux slogans, titres, intertitres), les discours ne sont pas pour autant interchangeables. Ainsi, là où en nous situant au niveau de procédés discursifs, nous trouvons un effacement des différences au profit du consensuel, une analyse lexicométrique fine des mêmes prospectus peut retrouver une répartition cohérente avec l'échiquier politique. (Ibidem, p. 13)

1. H. Constantin de Chanay, 2004, «Des couleurs incolores? L'épidictique dans les prospectus

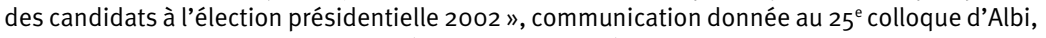
«Langages et signification », consacré en 2004 aux « Rhétoriques des discours politiques ».

IEP de Lyon, Médias et identités, jean-michel.rampon@univ-lyon2.fr 
Il s'avère qu'en marge d'une journée d'étude de la Société des Amis de Mots, qui s'est tenue à Lyon en octobre 2002, j'ai eu l'occasion de présenter une photographie des distances entre les seize candidats à l'élection présidentielle française de la même année et ce, à l'aune d'une approche lexicométrique des professions de foi adressées aux électeurs quelques jours avant le 21 avril, date du premier tour ${ }^{2}$. L'analyse arborée (fig. 1, infra) donne à voir d'une certaine manière une répartition cohérente avec l'échiquier politique ${ }^{3}$. Elle permet d'entériner certains rapprochements : ainsi de Bruno Mégret et de Jean-Marie Le Pen à l'extrême droite, malgré une certaine persistance de leur part à laisser accroire qu'il n'en est rien, mais aussi d'Arlette Laguiller, Daniel Gluckstein et Olivier Besancenot à l'extrême gauche. Une nuance de taille concerne cependant ces derniers (O. Besancenot dans une moindre mesure) : c'est la distance qui les sépare de l'ensemble des autres candidats - ceux de l'extrême droite compris -, ce qui est la marque d'un vocabulaire original, ou pour le moins caractéristique. L'analyse arborée présentée infra autorise encore une qualification de certains autres regroupements autour d'un même nœud ou de nœuds proches, sur la base de ce qui peut être connu des candidats. Par exemple, comment ne pas être tenté d'apprécier le bouquet composé de Noël Mamère, Corine Lepage et Christiane Taubira à l'aune de l'étiquette "petits » candidats (ainsi qu'ils ont été désignés parfois par une partie des médias) et le duo Jean-Pierre Chevènement/François Bayrou via leur autodésignation comme «troisième candidat » (plus encore avérée pour le premier) ? Ce que révèle également cette analyse, c'est la position relativement isolée de Jean Saint-Josse. Enfin, il est un rapprochement susceptible de donner lieu à glose, c'est celui de Jacques Chirac avec Lionel Jospin. Cette proximité peut-elle s'expliquer par leur profil commun, en tout cas au moment où les professions de foi sont diffusées, de possible futur président ? Bien sûr, il serait légitime de chercher à savoir au (plus) juste quels termes en particulier sont au fondement d'une telle proximité. Pour autant, et faute de place, j’ai préféré présenter ici quelques résultats obtenus à partir de ce qu'il conviendrait d'appeler une série d'intuitions exploratoires.

2. Précisons que c'est lors de cette même journée d'étude que H. Constantin de Chanay a présenté les tout premiers résultats de son test. Rappelons ici quels étaient les seize candidats en lice : François Bayrou, Olivier Besancenot, Christine Boutin, Jean-Pierre Chevènement, Jacques Chirac, Daniel Gluckstein, Robert Hue, Lionel Jospin, Arlette Laguiller, Corine Lepage, Jean-Marie Le Pen, Alain Madelin, Noël Mamère, Bruno Mégret, Jean Saint-Josse et Christiane Taubira.

3. Ce n'est pas le lieu ici de présenter les mesures qui sous-tendent l'analyse arborée. Le lecteur intéressé par cette technique de classification pourra se reporter utilement à l'article que son concepteur Xuan Luong a corédigé avec Damon Mayaffre dans la revue Histoire \& Mesure (2003) et à la présentation qu'en fait Étienne Brunet dans les versions les plus récentes de son logiciel Hyperbase - utilisé ici -, lequel intègre désormais cette technique. 


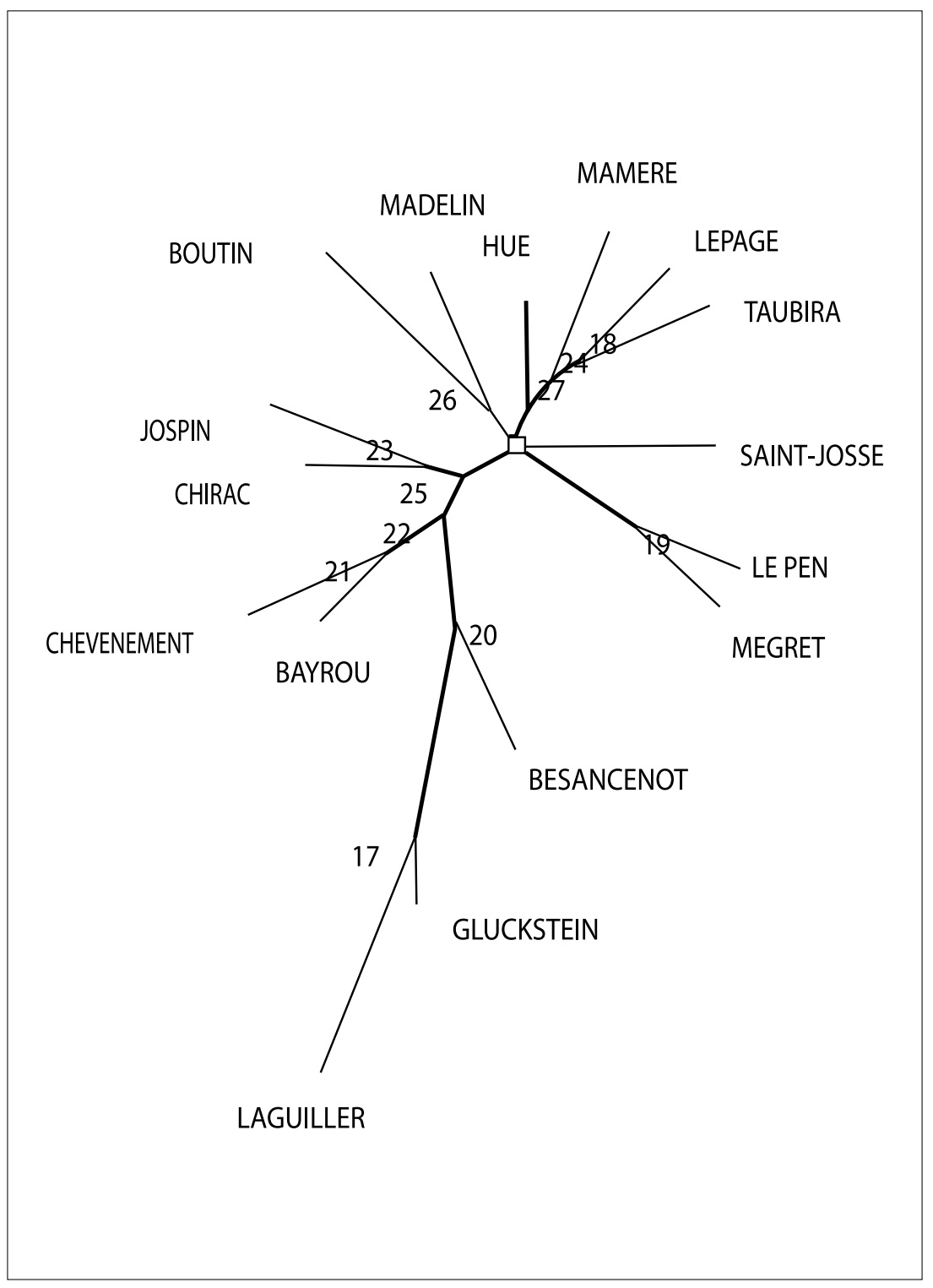

Figure 1 : Analyse arborée de la distance lexicale calculée sur les fréquences ${ }^{4}$ (corpus Professions de foi - Élection présidentielle 2002). Représentation radiale.

4. Pour chaque terme est appréciée la distribution réelle des fréquences dans toutes les combinaisons de textes pris deux à deux, sur l'ensemble du vocabulaire et sans seuil de fréquence préétabli. 


\section{Objectif et démarche de l'analyse}

On sait que l'analyse lexicométrique de textes politiques met assez généralement l'accent sur des termes habités, à tout le moins qui équivalent à des modes différents de désignation et de référenciation d'une même réalité sociopolitique (la société française en 2002, par exemple), ou encore qui prennent sens à condition d'apprécier au plus juste l'interdiscours qui les caractérise. Pour autant, l'hypothèse que je voudrais défendre ici est que certains verbes peuvent fonctionner comme des indicateurs de véritables lignes de partage entre candidats. Un même verbe peut être surreprésenté chez un seul candidat et alors la spécificité d'emploi est à même de révéler un point de fixation du discours de ce candidat ${ }^{5}$. Mais un même verbe peut aussi être privilégié non pas chez un seul mais chez deux ou plusieurs candidats. L'intérêt peut être alors de déceler des proximités verbales entre candidats. Dans cet ordre d'idée, la valeur d'un tel rapprochement sera d'autant plus forte que les candidats qui en sont partie prenante sont proches sur l'échiquier politique dont il a été question en amont. II n'est pas question ici de s'interroger sur la pertinence ou le bienfondé d'un tel système de places sur le spectre politique français en 2002 (par exemple, par rapport à celui mis en valeur par l'historien Antoine Prost à la fin du $19^{\mathrm{e}}$ siècle, déjà à partir de professions de foi, autrement dit au moment où le concept de classe politique se consolide), mais plutôt d'entériner ces positionnements comme réalité objectivée sur le plan de la communication médiatico-politique. Dans le présent article, je vais privilégier deux aspects liés aux verbes, sans que le corpus constitué ne puisse être au final limité à eux:

- D'abord, une photographie des distances entre candidats sur la base des verbes à l'infinitif effectivement utilisés dans les professions de foi qui nous occupent ici. Faire miser une première approche sur les seuls verbes à l'infinitif ne relève pas du hasard. Il ne faut pas oublier en effet que le genre profession de foi correspond avant tout à un programme qui décline des propositions sur un mode énumératif ${ }^{6}$. Une telle présentation favorise le recours à l'infinitif. Mon hypothèse est en tout cas que ces formes verbales, une fois isolées, vont déjà produire quelques informations sur les registres de l'agir prônés par les différents candidats et sur d'éventuels rapprochements partisans (ou fidèles à une répartition par grandes familles sur l'échiquier politique). Pour ce faire, j'ai pro-

5. Afin de ne pas alourdir le texte, ici, comme à chaque fois que la référence n'est pas directement nominative et se décline au masculin/féminin, le terme «candidat » désigne candidat et candidate.

6. À l'exception notable, généralement, des candidats d'extrême gauche. Parce que ces candidats savent qu'ils ne seront de toutes façons pas présents au second tour, ils proposent des professions de foi atypiques, y compris dans leur longueur. Ce que confirme le présent corpus: les professions de foi de D. Gluckstein et d'A. Laguiller, avec un nombre d'occurrences supérieur à 2500 , se situent aux deux premières places (mais celle d'O. Besancenot à la neuvième). 
cédé à une analyse factorielle des correspondances, en ne retenant que les formes verbales à l'infinitif enregistrées trois fois ou plus dans le corpus.

- Ensuite, une appréciation de la distance sur la base d'une approche mêlée des formes fléchies des verbes parmi les plus déclinés et des termes non verbaux qui leur sont associés, y compris via des éléments (je reviendrai sur ce terme) qui entrent dans la composition de l'ensemble de ces formes lexicales. D'une certaine façon, il serait possible de parler à leur égard de verbes « ordinaires ». En effet, quand on cherche à isoler les verbes qui comptent au moins seize occurrences, quel que soit par ailleurs le nombre de formes fléchies différentes pour chacun d'eux, on obtient la liste suivante, de laquelle ont été retranchés les auxiliaires être et avoir : faire, devoir, vouloir, pouvoir, falloir, donner, dire, aller, créer, défendre, permettre, voter, proposer, vivre, prendre, respecter, voir, garantir, favoriser, assurer, élire, savoir, refuser, augmenter, mettre et laisser ${ }^{7}$. Comme je l'ai dit, il m'a paru intéressant de tester la prégnance avérée de telles formes verbales au gré des candidats en élargissant l'assise, ou le socle, de l'analyse aux formes non verbales qu'il est possible de leur associer, y compris par l'entremise d'éléments qui sont à la base de la morphologie lexicale des unes et des autres. Pour ce faire, le dictionnaire Le Robert Brio m'a servi de guide dans la constitution des listes de termes apparentés ${ }^{8}$. Pourquoi mêler des termes qui n'ont en commun, au mieux, qu'un rapport morpho-lexical ? Précisément pour vérifier l'hypothèse selon laquelle il est possible d'associer à chaque candidat au moins une déclinaison lexicale sur la base du verbe comme mot-base. Une telle focalisation permettrait de mettre au jour une manière de permanence lexicale au gré des candidats, à titre individuel ou collectif, avec, dans ce dernier cas, la question en arrière-fond des rapprochements possibles entre candidats, selon le critère partisan.

7. Autrement dit, et de façon théorique, les seize occurrences peuvent correspondre à seize formes fléchies différentes ou, à l'extrême opposé, à une seule qui serait répétée seize fois à l'identique. Le nombre seize a été retenu parce que, là encore d'un point de vue théorique, il est possible d'imaginer une répartition aléatoire qui verrait une occurrence être attribuée à chacun des seize candidats. Mais il n'a pas de valeur significative autre que celle-là et on aurait pu tout aussi bien prendre un seuil plus bas.

8. On se reportera utilement à la préface (p. VII-XVII) qu'a rédigée Josette Rey-Debove pour cet ouvrage qui, en son édition de 2004 et avec des remaniements, a pris la suite du Robert méthodique et du Nouveau Robert méthodique. Voir en particulier cet extrait: "Nous avons appelé indifféremment éléments les affixes et les radicaux pour ne pas imposer une partition des deux types au détriment de l'intuition du lecteur. [...] Étant en position de distinguer la morphologie de l'étymologie, nous faisons la différence entre une famille étymologique (même étymon) et une famille lexicale (même morphème : morphème libre, qui est un mot, ou morphème lié - qui est un élément). » (p. X) Parler dans le présent article de formes dérivées serait sans doute apparu abusif dans la mesure où il arrive que des verbes aient été créés à partir de noms, par exemple, et se trouvent de fait en position de dérivés. C'est pourquoi j'ai préféré qualifier les termes d'apparentés, expression qui me parait assez fidèle avec ce qui est recherché ici : une proximité morpho-lexicale entre deux termes. 


\section{Première appréciation de la distance : les verbes à l'infinitif}

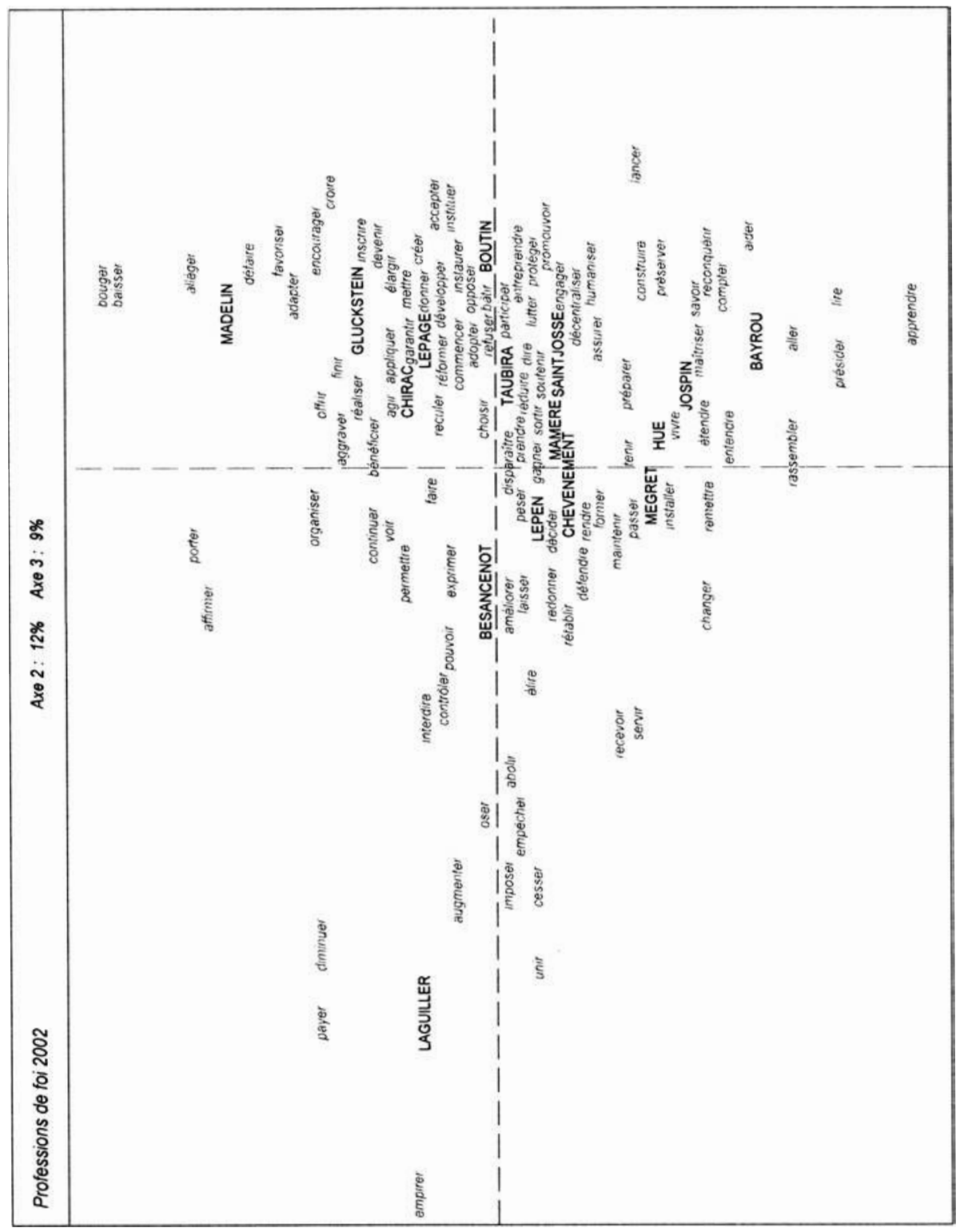

Figure 2

Analyse factorielle des correspondances (axes 2 et 3 ).

16 candidats $x$ verbes à l'infinitif $(F \geq 3)$ 
De l'analyse factorielle des correspondances produite à partir des verbes à l'infinitif, il ressort les constats suivants (voir fig. 2 supra) ${ }^{9}$ : plusieurs verbes entrent en concaténation en raison de leur filiation sémantique. Par exemple, pour ne mentionner que les plus longues chaines:

- encourager, commencer (quadrant supérieur droit), entreprendre, promouvoir, engager et lancer (quadrant inférieur droit), soit des verbes inchoatifs qui expriment un procès dans sa phase d'amorce ;

- devenir (quadrant supérieur droit) puis passer, rendre (pour l'essentiel ici dans le sens de "faire devenir »), améliorer et changer (ces quatre derniers verbes assez proches les uns des autres dans le quadrant inférieur gauche), soit des verbes qui désignent une évolution, un processus (souhaité en tout cas);

- garantir, protéger, préserver (en enfilade à l'intérieur des quadrants supérieur et inférieur droits), défendre et maintenir (placés à proximité dans le quadrant inférieur gauche), soit des verbes qui renvoient à l'idée de permanence d'un état ou d'un acquis ;

- réaliser, créer, bâtir et construire (en arc de cercle de part et d'autre du premier axe, à droite du plan), soit des verbes de «faire » par excellence (interchangeables du reste avec l'infinitif faire);

- inscrire, instituer, instaurer (remarquablement proches dans le quadrant supérieur droit) et installer (situé à l'écart dans le quadrant inférieur gauche), soit des verbes qui vont de pair avec l'idée d'établir quelque chose ou quelqu'un dans la durée;

- enfin élargir, développer (eux aussi proches dans le quadrant supérieur droit) et étendre (quadrant inférieur droit), soit des verbes qui contiennent le trait du déploiement.

À cette liste, il serait encore possible d'ajouter les quatre verbes ayant en commun l'élément re- à l'initiale, tous présents dans le quadrant inférieur gauche : redonner, rétablir, remettre et rassembler. Bien que non réductibles aux candidats d'extrême droite, il est à noter que ces verbes, à la base de présupposés, ont la particularité de s'inscrire chez eux dans des énoncés récurrents ${ }^{10}$.

Sur la base d'un tel repérage, il m'a paru intéressant d'essayer de déterminer s'il y avait ou non des séries de verbes plus polarisées que d'autres (ce que la vue d'ensemble de l'analyse factorielle des correspondances ne permet pas toujours d'apprécier au plus près). Il apparait ainsi que les verbes qui se rapportent à l'idée de permanence d'un état ou d'un acquis sont les mieux

9. Il s'agit d'une représentation sur les axes 2 et 3 dans la mesure où le premier axe ne rendait guère visible que l'opposition entre D. Gluckstein, d'une part, et le maelström de tous les autres candidats, d'autre part.

10. «Redonner la parole au Peuple », « rétablir l'ordre et la loi » (.-M. Le Pen) et « remettre de l'ordre en France » (variante : « remettre notre pays à l'endroit »), « rassembler » autour de sa candidature (B. Mégret). 
partagés, jusque par dix des seize candidats pour défendre. Il faut sans doute voir là ce qui pourrait constituer le registre banal du vocabulaire de la profession de foi comme genre : répercuter des mesures ou des actions que l'on considère comme bonnes parce que déjà mises en application (ou applicables) et chercher à convaincre de leur bien-fondé. À l'inverse, trois des quatre verbes qui relèvent de l' « établir », et plus particulièrement ceux dont a été mentionnée la forte proximité, sont privilégiés du côté droit de l'échiquier (de façon totale pour instituer, quasi totale pour instaurer et inscrire). Or, dans le détail, il s'avère que ces verbes à l'infinitif sont pour une grande part utilisés par C. Boutin et, dans une moindre mesure, par C. Lepage. Il parait difficile, dans ces conditions, d'en déduire quelque chose, sauf à admettre éventuellement un effet de style. Mais parce que les deux verbes inchoatifs polarisés à droite que sont lancer et encourager sont également (surtout le premier) décelables dans la profession de foi de C. Boutin, il serait légitime de se demander dans quelle mesure ce qui peut être perçu comme une marque d'écriture n'est pas avant tout mis au service d'un combat politique ${ }^{11}$.

Contrairement aux verbes qui viennent d'être passés en revue, trop dépendants d'une seule candidate, les verbes de «faire » sont pour une grande part caractéristiques de candidats de droite, et selon des effectifs autrement plus fournis. Ce qui permet de s'interroger in fine sur la valence marquée à droite de tels verbes et de se risquer à affirmer à leur sujet qu'on a affaire à des verbes "de droite ». Si, au vu des contextes, une telle affirmation est discutable pour réaliser, elle semble moins sujette à caution avec bâtir et construire tant ces verbes servent à entremêler, chez un certain nombre de candidats de droite, avenir et communautés symboliques d'appartenance, au premier rang desquelles la nation et l'Europe.

Du côté gauche de l'échiquier, il n'y a guère que les séries de verbes caractérisées par la marque du déploiement et celle de l'évolution qui comportent une trace de polarisation, avec étendre et passer. Pour autant, il est difficile d'affirmer à ce stade que l'on a affaire à des formes verbales « de gauche ». Tout juste est-il possible de signaler que ces deux verbes servent à appuyer des revendications qui, elles, sont clairement situables à gauche ${ }^{12}$. En fait, les verbes qu'il serait possible de qualifier «de gauche » sont sans conteste ceux qui longent le premier axe dans sa partie la plus excentrée et qui se trouvent en forte oppo-

11. Comme le suggèrent les énoncés suivants (parmi d’autres) : «instituer contre la violence un "pacte de responsabilité familles-médias" ", "instaurer une démarche volontaire et solennelle d'obtention de la nationalité française », «inscrire dans notre Constitution le principe du respect de toute vie humaine de la conception à la mort naturelle », « lancer une grande campagne d'information contre le cannabis et toutes les drogues "...

12. "Étendre les services publics» (R. Hue), «étendre le pouvoir du Parlement » (N. Mamère) ; «la protection de la nature, des hommes et des femmes doit passer avant la course aux profits» (O. Besancenot), «création du droit de passer, tout au long de la vie, d'un emploi stable à un autre emploi » (R. Hue), « passer à la Sixième République » (N. Mamère). 
sition - dans l'espace du plan mais aussi d'un point de vue sémantique - avec certains des verbes déjà évoqués. Ainsi d'abolir (auquel on pourrait associer le verbe cesser voisin) avec instaurer ou instituer ${ }^{13}$ ou encore d'interdire avec favoriser, verbe uniquement employé par des candidats situés à droite de l'échiquier politique, A. Madelin en tête.

Parmi les formes qui favorisent l'allongement des axes se trouvent celles qui correspondent à une manière d'hapax, autrement dit les verbes à l'infinitif propres à un seul candidat: empirer (A. Laguiller), défaire (D. Gluckstein), apprendre (F. Bayrou), présider (L. Jospin), bouger et baisser (A. Madelin). Ce qu'il y a de remarquable, c'est que ces spécificités d'emploi se doublent à chaque fois d'une portée du discours qui confine à l'antienne : la condition des travailleurs (ou la situation du monde du travail) chez A. Laguiller, le traité de Maastricht chez D. Gluckstein, l'apprentissage de la lecture chez F. Bayrou, le mode de présidence chez L. Jospin, enfin la France et les impôts chez A. Madelin. Chez ces deux derniers candidats, le recours à un adverbe modalise fortement la portée du discours tenu : « présider autrement », « faire (enfin) bouger la France », «baisser (vraiment) les impôts». On pourrait se demander jusqu'à quel point le verbe à l'infinitif caractéristique de chacun des candidats n'a pas finalement pour fonction d'offrir un raccourci saisissant du contenu de sa profession de foi, sous forme d'hexis ou de posture morale.

\section{Deuxième appréciation de la distance : verbes fréquents et termes apparentés}

A déjà été présentée en amont la liste des verbes les plus fréquents. Je rappelle que j'ai eu recours au Robert Brio pour mettre au jour les formes qui se trouvent dans un rapport morpho-lexical évident avec ces verbes. Pour le présenter dans les termes mêmes du dictionnaire consulté, deux items vont être considérés comme appartenant à une même famille lexicale dès lors qu'ils comportent un même morphème, voire un morphème lié ou élément (voir note 7$)^{14}$. À partir d'une telle liste élargie, j'ai procédé au calcul d'un taux d'amplitude par candidat des formes verbales et de leurs termes apparentés. Pour un candidat donné, celui-ci est obtenu en divisant le nombre de formes différentes d'un même mot-base chez ce candidat par le nombre total de formes différentes de ce mot-base à l'échelle du corpus. Ce qui est en jeu, ce ne sont donc pas les fréquences partielle et totale des formes déclinables depuis un même

13. "Abolir la dette» (O. Besancenot), «abolir le secret bancaire » (A. Laguiller), « abolir la double peine » (N. Mamère).

14. Dans certains cas limités, j'ai choisi d'y ajouter des items qui se situent d'une certaine manière à la frontière entre morphologie et étymologie (ainsi des termes possible, possibilité et possibilités repérés dans le corpus, en ligne directe avec posse, « pouvoir »). 
mot-base, mais bien le nombre de formes différentes qui s'y rattachent, ne seraient-elles avérées qu'une seule fois dans le corpus. Comme je n'ai procédé à aucun effet de pondération en dépit des tailles différentes des professions de foi, j'ai retenu arbitrairement le nombre de huit formes différentes au total. Dans ces conditions, des verbes comme falloir (duquel n'est de toute façon dérivé aucun terme apparenté), laisser et augmenter ont été rejetés. Plus le résultat se rapproche de 1 , plus il correspond à un emploi marqué de formes différentes pour un même candidat ${ }^{15}$. Afin de ne pas alourdir la liste, n'ont été retenus pour chaque mot-base et par candidat que les taux d'amplitude supérieurs ou égaux à 0,33 . On trouvera dans le tableau 1 (voir en annexe) les résultats obtenus sur la base d'un tel protocole avec, pour chaque verbe comme mot-base, la liste des formes associées ${ }^{16}$.

Au vu des résultats, il faut bien reconnaitre une certaine déception. Aucune des séries ici mises au jour ne permet de déceler un effet de polarisation au-delà de deux candidats d'un même "bord ». Et même lorsqu'un tel rapprochement est avéré, il met surtout aux prises A. Laguiller et D. Gluckstein à quatre reprises, avec élire, voir, dire et faire. Sur la partie droite de l'échiquier, tout juste est-il possible de relever la proximité de J. Chirac et d'A. Madelin via les déclinaisons de formes liées à assurer ${ }^{17}$. S'il existe une sorte d'alliance objective à l'extrême gauche, elle se retrouve donc dans l'usage de verbes de base qui, pour cette raison même, sont les prototypes des catégories de verbes au sein desquelles ils prennent place : faire (action), dire (communication) et voir (perception). Avec élire, A. Laguiller et D. Gluckstein se montrent pareillement disposés à mettre en garde ceux à qui ils s'adressent sur la légitimité d'une telle élection et à affirmer que les véritables enjeux se trouvent ailleurs («Une majorité à la Chambre peut n'avoir été élue que par une minorité d'électeurs», proclame ainsi A. Laguiller). À un autre niveau, un rapprochement entre J.-M. Le Pen et B. Mégret est bien avéré avec défendre, mais leur sont associés F. Bayrou et R. Hue. Et à considérer les formes lexicales en contexte, l'alliance objective évoquée en amont pour deux des représentants de l'extrême gauche ne va pas de soi ici. En effet, si B. Mégret dit de lui-même qu'il « défend l'ordre républicain » et qu'il a pour objectif de «défendre les Français », ainsi que l’on pouvait s'y attendre,

15. Dans le cas où il serait égal à 1 (cas qui ne se présente pas ici), cela signifierait que toutes les déclinaisons d'un mot-base avérées dans le corpus se trouveraient présentes chez un même candidat.

16. Chaque liste, et donc la mesure qu'elle autorise, a été obtenue après désambigüisation, i.e. distinction pour l'essentiel des formes adjectivales et nominales qui pouvaient apparaitre hors contexte comme des participes passés, mais aussi dégroupage des homographes renvoyant à des réalités lexicales différentes («pouvoir » $[v][n]$, « faites » [indicatif][part. passé]...).

17. Si A. Madelin est favorable à une désétatisation de l'assurance-maladie et à la création d'une assurance-dépendance, J. Chirac envisage une assurance-emploi. À cet égard, celui-ci montre son attachement au système de retraite par répartition, " qui assure la solidarité entre tous les Français », tandis qu'A. Madelin souhaite voir «assurer l'équité des retraites, notamment entre le privé et le public ». 
la déclinaison majeure de défendre qu'effectue J.-M. Le Pen dans sa profession de foi est d'une tout autre portée, ainsi que le laisse percevoir l'énoncé suivant: «Défendre la nature et la vie en faisant à nouveau triompher la vie sur la culture de mort et en défendant la véritable écologie». Sans doute serait-il possible encore de mettre l'accent sur un duo particulier, celui de C. Boutin et de C. Taubira. Dans la partie basse du tableau où sont confinés les items auxquels sont rattachés trois candidats, ces deux candidates se retrouvent ensemble trois fois sur quatre. Si l'on met de côté permettre et respecter pour se focaliser sur devoir, il est possible de réintroduire l'idée d'une forme d'alliance objective, à droite cette fois. En effet, J. Chirac et C. Boutin associent de façon récurrente à devoir la France et l'État. L'usage du nous et du vous leur sert tout à la fois à interpeller les électeurs et à les intégrer dans le programme ainsi dessiné, programme que symbolise à lui seul chez C. Boutin la «Charte des Devoirs de l'Homme ». À l'opposé, et bien qu'avec un taux d'amplitude identique à celui de C. Boutin $(0,38), C$. Taubira articule les cinq formes différentes de devoir qu'elle convoque dans son discours à une vingtaine de référents différents, soit quasiment un nouveau à chaque nouvelle occurrence qui apparait au fur et à mesure que l'on progresse dans sa profession de foi. Ainsi, et pour ne s'en tenir qu'aux termes politique et République: «la politique éducative devra consolider le socle des valeurs républicaines », "les politiques de santé, donc de prévention, doivent être privilégiées sur les politiques de la maladie », « la culture et la politique doivent primer l'économie et la technique ", " elle [une politique de confiance sociale] doit être fondée sur les principes républicains et radicaux de laïcité, d'humanisme et d'universalisme », « les principes de la République doivent marquer la construction de l'Europe fédérale », «la Sixième République doit reposer sur une Constitution qui consacre une réelle séparation des pouvoirs ».

Enfin, s'il fallait un exemple parmi d'autres de ce que les formes lexicales liées à vouloir représentent pour ainsi dire un faisceau de termes servant à unifier formellement les discours du genre profession de foi, on pourrait le trouver dans la mise en miroir suivante :

\begin{tabular}{|l|l|}
\hline \multicolumn{1}{|c|}{ Bruno Mégret } & \multicolumn{1}{c|}{ Lionel Jospin } \\
\hline $\begin{array}{l}\text { Je veux remettre de l'ordre en France. } \\
\text { la volonté de rassembler }\end{array}$ & $\begin{array}{l}\text { Je veux une France plus juste. } \\
{[\ldots] \text { nous pouvons trouver des réponses, si }} \\
\text { nous savons nous rassembler autour d'un } \\
\text { projet solide, d'une volonté ferme }[\ldots . .]\end{array}$ \\
$\begin{array}{l}{[\ldots] \text { je voudrais vous adresser un message }} \\
\text { d'espoir pour vous dire }[\ldots]\end{array}$ & $\begin{array}{l}{[. . .] \text { je voudrais vous dire la conviction qui }} \\
\text { m'anime plus que jamais. }\end{array}$ \\
\hline
\end{tabular}


Finalement, l'intérêt majeur provient des items qui ne se trouvent associés au final qu'à un seul candidat. Sans restreindre, bien évidemment, les professions de foi où ils prennent place à leur présence spécifique, il semble malgré tout opportun de se demander jusqu'à quel point ils n'en caractérisent pas un trait majeur, voire une direction globale, sinon une posture morale, ainsi que précisé en amont.

C'est ainsi que D. Gluckstein inscrit (re)mettre dans des expressions plus larges qui, presque toutes, servent à présenter un phénomène ou un processus sous un jour péjoratif, sinon négatif - mettre à l'ordre du jour, mettre en place (2), mettre en œuvre et remettre en cause (2), tournure présupposée qui entre en écho avec les trois occurrences de refaire qu'utilise par ailleurs ce candidat. Dans la mesure où le verbe infinitif qui lui est spécifique, ainsi qu'il nous a été donné de le voir antérieurement, est défaire, on a là un faisceau d'indices non dénué d'intérêt quant à une construction du monde qui passe chez ce candidat par un usage marqué de préfixes à même de transformer le sens premier des termes auxquels ils se trouvent accolés.

En entrecroisant certains des énoncés qui contiennent créer et refuser, il est possible d'opérer un nouveau rapprochement thématique entre J. Chirac et A. Madelin. Pour autant, leur position n'est pas la même et là où le discours du président sortant dénote projet et projection, celui d'A. Madelin consiste prioritairement à donner de lui l'image d'un homme aux convictions fortes et qui n'est prêt à aucune concession : « Les entreprises, et tous ceux qui créent l'emploi, doivent voir allégées les contraintes fiscales et administratives qui pénalisent leur développement par rapport à leurs concurrents européens » (J. Chirac) ; "Il prépare une grande réforme fiscale et la réforme de notre protection sociale. Il se heurte au refus du Premier ministre. Fidèle à ses engagements, il quitte le gouvernement. » (A. Madelin)

L. Jospin, quant à lui, se distingue par des formes lexicales dans la lignée de garantir. Si ce terme est en particulier articulé avec les « retraites » (par répartition), il permet aussi à L. Jospin d'exprimer sa vision "rénovée » du socialisme et d'un chef d'État s'en prévalant aujourd'hui, vision partagée ailleurs en Europe et dont on sait qu'elle fait débat à gauche : « Le président de la République doit être (...) un président qui est le garant d'une société où le progrès économique et le progrès social vont de pair, où il est aussi important de produire que de redistribuer, où l'égalité des chances et l'innovation avancent ensemble. " Son absence au second tour a littéralement fait voler en éclats les termes du contrat moral passé avec les électeurs et dont le socle aura cherché à mixer la distinction («Présider autrement ») et la garantie par anticipation.

En ce qui concerne deux des items modaux présents à ce stade - savoir et pouvoir -, leur amplitude significative chez, respectivement, J.-P. Chevènement et $\mathrm{A}$. Laguiller relève pour ainsi dire d'une démarche contraire quant à la posture qu'ils se donnent. C'est ainsi que les différentes formes liées à savoir 
semblent comme s'imbriquer au gré du déroulement de la profession de foi de J.-P. Chevènement, au point de donner de lui l'image de l'homme de la situation, précisément parce qu'il sait ce qui est bon pour la France : «Vous le savez bien, la France ne sait plus où elle va »; «Vous savez que j'ai toujours fait ce que j'ai dit »; "L'école sera rappelée à ses missions: transmettre les savoirs et les valeurs; former des citoyens. » Avec pouvoir, A. Laguiller exprime le plus souvent des idées similaires entre elles, ce qui ne l'empêche pas d'avoir recours à un grand nombre de formes différentes (11 pour 17 occurrences au total) comme dans : «Cela je ne pourrai pas le faire sans vous » (futur), à comparer, par exemple, avec: «Et même si j'étais élue, je ne pourrais rien sans le soutien actif du monde du travail» (conditionnel).

Terminons avec J. Saint-Josse. Si l'élément vital prédomine chez le représentant du parti Chasse, pêche, nature et traditions, il n'en confine pas moins à une forme d'antienne qui lui est caractéristique - « qualité de vie » (3), «bassin de vie », «modes de vie», «bien vivre», « le tissu vivant et convivial de nos villages », « revitaliser le monde rural »...

Comme toute analyse lexicométrique, la présente étude n'épuise pas le contenu des professions de foi de 2002 puisqu'elle s'appuie pour l'essentiel sur les verbes. Leur déclinaison à l'infinitif a permis de mettre au jour certaines spécificités d'emploi marquées du point de vue de leur répartition sur l'échiquier politique, dans sa conception traditionnelle (ainsi de bâtir, construire ou favoriser à droite et de cesser, abolir et interdire à gauche). Ces professions de foi auront également représenté un prétexte pour tester un taux d'amplitude à partir des verbes les plus fréquents et des termes qui leur sont apparentés. L'idée était de mettre au jour des permanences lexicales au gré des candidats mais aussi, au-delà, des regroupements partisans. Sur ce dernier point, il faut bien admettre une certaine déception par rapport à ce qui était attendu, dans la mesure où aucun effet de polarisation n'est perceptible au-delà de deux candidats. Ces résultats restent cependant amendables puisqu'ils ont été obtenus à partir d'une méthode, volontairement présentée ici dans une phase d'intuition exploratoire, qui gagnerait à être précisée plus avant. De façon générale, un élargissement à d'autres professions de foi liées à l'élection présidentielle française, antérieures ou... à venir, permettrait certainement d'affiner et l'approche, et les résultats dans leur ensemble. En fin de compte, l'intérêt me semble devoir être trouvé dans les séries de termes spécifiques à un seul candidat, dans ce qu'elles révèlent potentiellement d'un hexis ou d'une posture morale adopté(s) au moment de se présenter devant les électeurs. 


\section{Références}

BONHOMMEM., 2004, "Monologisme et dialogisme dans les professions de foi électorales: le cas des élections présidentielles françaises de 2002 », Structures et discours. Mélanges offerts à Eddy Roulet, A. Auchlin et al. éd., Québec, Nota bene, p. 349-365.

LUONGX., MAYAFFRE D., 2003, "Les discours de Jacques Chirac (1995-2002). Représentation arborée et généalogie politique », Histoire \& Mesure, vol. 18, $n^{\circ} 3 / 4$, p. 289-311.

\section{Annexe}

\begin{tabular}{|c|c|c|}
\hline Mot-base & Termes apparentés $(\mathrm{F} \geq 1)$ & $\begin{array}{l}\text { Candidat-e concerné-e } \\
\text { (taux d'amplitude } \geq 0,33 \text { ) }\end{array}$ \\
\hline Pouvoir & $\begin{array}{l}\text { contre-pouvoir, peut, peut-être, peuvent, pos- } \\
\text { sibilité, possibilités, possible, pourra, pourrai, } \\
\text { pourrais, pourrait, pourront, pouvait, pouvez, } \\
\text { pouvoir [v.], pouvoir [n.], pouvons, pu, puis- } \\
\text { sance, puissant, puisse, puissent, puissiez }\end{array}$ & Laguiller $(0,43)$ \\
\hline (Re)mettre & $\begin{array}{l}\text { mettent, mettons, mettrai, mettre, mis, mise, } \\
\text { mise [n.], remet, remettant, remettons, remet- } \\
\text { tre, remis, remise [n.] }\end{array}$ & Gluckstein $(0,38)$ \\
\hline Refuser & $\begin{array}{l}\text { refus, refusant, refuse, refusent, refuser, } \\
\text { refusé, refusée, refusons }\end{array}$ & Madelin $(0,37)$ \\
\hline Garantir & $\begin{array}{l}\text { garant, garanti, garanti [adj.], garantie, garan- } \\
\text { tie [n.], garanties [adj.], garanties [n.], garantir, } \\
\text { garantis, garantissant, garantisse }\end{array}$ & Jospin $(0,36)$ \\
\hline Vivre & $\begin{array}{l}\text { revitalisation, revitaliser, survivre, vie, vies, } \\
\text { vital, vitales, vitalité, vitaux, vivant [n.], vivant } \\
\text { [adj.], vivante, vivantes, vive, vivent, vivre }\end{array}$ & Saint-Josse $(0,35)$ \\
\hline Créer & $\begin{array}{l}\text { créant, création, créations, créatures, crée, } \\
\text { créent, créer, créerai, créé, créée, créées, } \\
\text { recréer }\end{array}$ & Chirac $(0,33)$ \\
\hline Savoir & $\begin{array}{l}\text { sais, sait, saura, saurait, savez, savoir [v.], } \\
\text { savoir [n.], savoirs, savons }\end{array}$ & Chevènement $(0,33)$ \\
\hline \multirow[t]{2}{*}{ Elire } & $\begin{array}{l}\text { électeurs, élection, élections, électorale, } \\
\text { éligibilité, élire, élu, élu [n.], }\end{array}$ & Laguiller $(0,56)$ \\
\hline & $\begin{array}{l}\text { élu [adj.], élue, élue [n.], élus, élus [n.], élus } \\
\text { [adj.], réélire, réélue }\end{array}$ & Gluckstein $(0,37)$ \\
\hline
\end{tabular}




\begin{tabular}{|c|c|c|}
\hline Mot-base & Termes apparentés $(F \geq 1)$ & $\begin{array}{l}\text { Candidat-e concerné-e } \\
\text { (taux d'amplitude } \geq 0,33 \text { ) }\end{array}$ \\
\hline \multirow[t]{2}{*}{ Voir } & $\begin{array}{l}\text { verraient, verrons, voient, voir, voit, voyez, vu, } \\
\text { vue [n.], vue [adj.] }\end{array}$ & Laguiller $(0,55)$ \\
\hline & & Gluckstein $(0,44)$ \\
\hline \multirow[t]{2}{*}{ Dire } & $\begin{array}{l}\text { c'est-à-dire, dira, dirai, dire, direz, dis, di- } \\
\text { saient, dit [ind.], }\end{array}$ & Gluckstein $(0,5)$ \\
\hline & $\begin{array}{l}\text { dit [part.], dite [adj.], qu'en-dira-t-on, soi- } \\
\text { disant }\end{array}$ & Laguiller $(0,41)$ \\
\hline \multirow[t]{2}{*}{ Voter } & $\begin{array}{l}\text { votaient, votant, vote, vote [n.], votent, voter, } \\
\text { voterez, votez, }\end{array}$ & Besancenot $(0,5)$ \\
\hline & votons, votée & Mégret $(0,4)$ \\
\hline \multirow[t]{2}{*}{ Faire } & $\begin{array}{l}\text { défaire, faire, fais, faisant, faisons, fait [ind.], } \\
\text { fait [part.], fait [n.], }\end{array}$ & Laguiller $(0,44)$ \\
\hline & $\begin{array}{l}\text { faite, faite [adj.], faites [ind.], faites [part.], } \\
\text { faits [n.], ferai, ferait, ferez, ferons, font }\end{array}$ & Gluckstein $(0,33)$ \\
\hline \multirow[t]{2}{*}{ Favoriser } & $\begin{array}{l}\text { défavorisées, faveur, favorable, favorables, } \\
\text { favorisant, favorise, }\end{array}$ & Madelin $(0,44)$ \\
\hline & favorisent, favoriser, favorisera & Hue $(0,33)$ \\
\hline \multirow[t]{2}{*}{ Assurer } & $\begin{array}{l}\text { assurance-dépendance, assurance-emploi, } \\
\text { assurance-maladie, }\end{array}$ & Chirac $(0,36)$ \\
\hline & $\begin{array}{l}\text { assurances, assurant, assure, assurer, assu- } \\
\text { rera, assurés, assureurs, rassurés }\end{array}$ & Madelin $(0,36)$ \\
\hline \multirow[t]{2}{*}{ Donner } & $\begin{array}{l}\text { donnant, donne, donnée, données, donnent, } \\
\text { donner, donnerons, }\end{array}$ & Madelin $(0,33)$ \\
\hline & donnez, donnons, dons, redonne, redonner & Mégret $(0,33)$ \\
\hline \multirow[t]{2}{*}{ Proposer } & $\begin{array}{l}\text { propos, propose, proposent, proposer, propo- } \\
\text { serai, proposée, }\end{array}$ & $\begin{array}{l}\text { Gluckstein }(0,5) \\
\text { Boutin }(0,37)\end{array}$ \\
\hline & proposition, propositions & Chevènement $(0,37)$ \\
\hline Devoir & $\begin{array}{l}\text { devant, devez, devoir [n.], devoirs, devons, } \\
\text { devra, devraient, }\end{array}$ & Chirac $(0,46)$ \\
\hline
\end{tabular}




\begin{tabular}{|c|c|c|}
\hline Mot-base & Termes apparentés $(F \geq 1)$ & $\begin{array}{l}\text { Candidat-e concerné-e } \\
\text { (taux d'amplitude } \geq 0,33 \text { ) }\end{array}$ \\
\hline & devrait, devrez, devront, doit, doivent, due & $\begin{array}{l}\text { Boutin }(0,38) \\
\text { Taubira }(0,38)\end{array}$ \\
\hline \multirow[t]{2}{*}{ Respecter } & $\begin{array}{l}\text { respect, respectant, respecte, respectent, } \\
\text { respecter, respecterai, }\end{array}$ & Boutin $(0,45)$ \\
\hline & $\begin{array}{l}\text { respecté, respectée, respectés, respectueuse, } \\
\text { respectueux }\end{array}$ & $\begin{array}{l}\text { Taubira }(0,45) \\
\text { Hue }(0,36)\end{array}$ \\
\hline \multirow[t]{2}{*}{ Permettre } & $\begin{array}{l}\text { permet, permettant, permettent, permettons, } \\
\text { permettra, permettre, }\end{array}$ & $\begin{array}{l}\text { Boutin }(0,33) \\
\text { Gluckstein }(0,33)\end{array}$ \\
\hline & permettront, permis, permis [n.] & Taubira $(0,33)$ \\
\hline \multirow[t]{2}{*}{ Défendre } & $\begin{array}{l}\text { défend, défendant, défende, défendent, } \\
\text { défends, défendrai, }\end{array}$ & $\begin{array}{l}\text { Bayrou }(0,5) \\
\text { Hue }(0,37)\end{array}$ \\
\hline & défendre, défense & $\begin{array}{l}\text { Le Pen }(0,37) \\
\text { Mégret }(0,37)\end{array}$ \\
\hline \multirow[t]{4}{*}{ Vouloir } & $\begin{array}{l}\text { veulent, veut, veux, volonté, voudrais, vou- } \\
\text { laient, voulez, }\end{array}$ & $\begin{array}{l}\text { Bayrou }(0,44) \\
\text { Gluckstein }(0,44)\end{array}$ \\
\hline & voulons, voulu & $\begin{array}{l}\text { Hue }(0,44) \\
\text { Jospin }(0,44)\end{array}$ \\
\hline & & $\begin{array}{l}\text { Mégret }(0,44) \\
\text { Chevènement }(0,33)\end{array}$ \\
\hline & & Madelin $(0,33)$ \\
\hline
\end{tabular}

Tableau 1 : Taux d'amplitude $\geq 0,33$ par mot-base et par candidat avec déclinaison des formes différentes. 